\title{
APPROACH FOR IMPROVING THE INTEGRATED SENSOR ORIENTATION
}

\author{
Mitishita, E., Ercolin Filho, L., Graça, N., Centeno, J.
}

\begin{abstract}
Geodetic Sciences Graduate Program - Department of Geomatics - Federal University of Paraná, UFPR - Centro Politécnico - Setor de Ciências da Terra - CEP 81531-990 - Curitiba, Paraná, Brazil - mitishita@ufpr.br; leonardo.ercolin@ gmail.com; niarkiosnl@hotmail.com; centeno@ufpr.br
\end{abstract}

\section{Commission I/3}

KEY WORDS: Integrated Sensor Orientation, Direct Sensor Orientation, In Situ Calibration, Photogrammetry

\begin{abstract}
The direct determination of exterior orientation parameters (EOP) of aerial images via integration of the Inertial Measurement Unit (IMU) and GPS is often used in photogrammetric mapping nowadays. The accuracies of the EOP depend on the accurate parameters related to sensors mounting when the job is performed (offsets of the IMU relative to the projection centre and the angles of boresigth misalignment between the IMU and the photogrammetric coordinate system). In principle, when the EOP values do not achieve the required accuracies for the photogrammetric application, the approach, known as Integrated Sensor Orientation (ISO), is used to refine the direct EOP. ISO approach requires accurate Interior Orientation Parameters (IOP) and standard deviation of the EOP under flight condition. This paper investigates the feasibility of use the in situ camera calibration to obtain these requirements. The camera calibration uses a small sub block of images, extracted from the entire block. A digital Vexcel UltraCam XP camera connected to APPLANIX POS AV ${ }^{\mathrm{TM}}$ system was used to get two small blocks of images that were use in this study. The blocks have different flight heights and opposite flight directions. The proposed methodology improved significantly the vertical and horizontal accuracies of the 3D point intersection. Using a minimum set of control points, the horizontal and vertical accuracies achieved nearly one image pixel of resolution on the ground (GSD). The experimental results are shown and discussed.
\end{abstract}

\section{INTRODUCTION}

The determination of exterior orientation parameters (EOP) is a fundamental work phase in all photogrammetric projects related to geoinformation extraction from imagery. Nowadays, the majority of the EOP determination is performed by the integration of the Inertial Measurement Unit (IMU) and GPS using two basic approaches. In the first, known as Direct Sensor Orientation, the EOP are directly computed by the GPS/INS measurements. The second, known as Integrated Sensor Orientation, is used to refine the direct EOP when their values do not achieve the required accuracies for the photogrammetric application. In this case, the direct EOP values are used as additional observation in the bundle block adjustment. The performance, requirements, advantage and disadvantage of the two approaches were are well studied and discussed in many research articles (e.g. Colomina 1999; Cramer 1999; Toth 1999; Heipke et al., 2002; Cramer \& Stallmann, 2002; Yastikli and Jacobsen, 2005; Ip et al., 2007).

The accurate computation of 3D coordinates by photogrammetric intersection based on Direct Sensor Orientation, due to an extrapolation process (Jacobsen and Wegmann, 2002), requires accurate Interior Orientation Parameters (IOP) and Exterior Orientation Parameters. To estimated accurate EOP from direct sensor orientation it is required accurate parameters related to sensors mounting (the offsets of the IMU relative to the projection centre and the angles of boresigth misalignment between the IMU and the photogrammetric coordinate system) (Yastikli and Jacobsen, 2005). Many researchers in the world (Jacobsen, 2000; Heipke et al., 2002; Cramer and Stallman, 2002; Wegmann, H., 2002; Honkavaara et al., 2003) investigated the stability of the geometric relationship of the IMU to the imaging system and the stability of the interior orientation. From the obtained results, it was concluded that the interior orientation and the mounting parameters can vary over time. For instance, the interior orientation parameters can change under flight conditions due to the effects of temperature and pressure (Yastikli and Jacobsen, 2005). Then, to improve the accuracy of the Direct Georeferecing, the system calibration, including the interior orientation and mounting parameters, is recommended before or after each photogrammetric mission.

The system calibration, in principle, can be performed before or after every mission to check the quality of the mounting and IOP. However, due to technical requirements, time and cost, the system calibration is not regularly applied. Then, the Integrated Sensor Orientation (ISO) is used to improve the quality of the direct EOP estimation when its values do not attain the required accuracies for the photogrammetric application. However, to carry out the ISO with a minimum number of known ground control points over the area of the block of images or without any use of ground control points, three basic conditions must be met. First, the block configuration should have sufficient forward and side overlap areas with a minimum number of tie points in these areas (Von Gruber positions); second, accurate IOP values and third, accurate standard deviation for the direct EOP values (Cramer and Stallman, 2001). This paper focuses on the second and third requirements. A study was performed to figure out a methodology to improve the performance of the ISO approach based on the hypothesis that IOP estimated under flight condition improve the quality of ISO.

The following three sections contain information about the photogrammetric blocks used in this study, methodology to improve the accuracies of the Integrated Sensor Orientation, the obtained results from the performed experiments and discussed, 
as well as the conclusions and recommendations for future work.

\section{MATERIALS AND METHODOLOGY}

\subsection{Imaging sensor}

Images were obtained using a digital large format frame camera Vexcel UltraCamXp. The source image has 17310 pixels cross track and 11310 pixels along track. The physical pixel size is equal to 6 microns. The nominal focal length is equal to $100.500 \mathrm{~mm} \pm 0.002 \mathrm{~mm}$. Principal point coordinates $\left(\mathbf{x}_{\mathbf{p}}=-\right.$ $0.120 \mathrm{~mm} \pm 0.002 \mathrm{~mm}$ and $\left.\mathbf{y}_{\mathbf{p}}=0.0 \mathrm{~mm} \pm 0.002 \mathrm{~mm}\right)$. The remaining lens distortion is less than $0.002 \mathrm{~mm}$. The IMU equipment connected to the camera is the Applanix POSTrack AV 510 IMU. The IMU absolute accuracies (RMS) - Position < $0.1 \mathrm{~m}$; Roll and Pitch $<0.005 \mathrm{deg}$; Yaw $<0.008 \mathrm{deg}$.

\subsection{Blocks of images}

Two blocks of images were used in this study. The first block has 15 images in 3 strips. The strips were flown in the following directions: two in N-S, one in S-N. Each strip has about 5 images, acquired with nearly $60 \%$ and $30 \%$ of forward and side overlaps respectively. The flight height was approximately 1600 $\mathrm{m}$. The ground sample distance (GSD) for this flight height is close to $10 \mathrm{~cm}$. The second block has 11 images in 2 strips. One strip with 5 images was flown in W-E and another with 6 images was flown in E-W. The strips have nearly $60 \%$ and $30 \%$ of forward and side overlaps respectively. The flight height was approximately $2100 \mathrm{~m}$. The ground sample distance (GSD) for this flight height is close to $13 \mathrm{~cm}$. The project area has 18 signalized control points. The Figure 1 shows the layouts of the first and second blocks and the positions of the control points in each block. The three-dimensional coordinates of the 18 points were acquired by precise differential GPS survey. The root mean square error (RMSE) values for the $\mathrm{X}, \mathrm{Y}$ and $\mathrm{Z}$ coordinates were approximately $2 \mathrm{~cm}$.

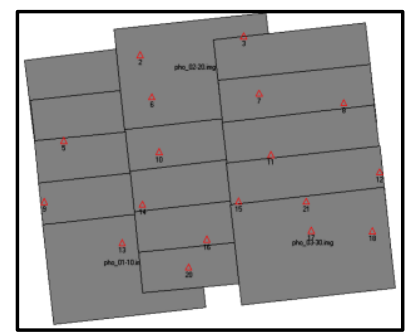

(a) - First Block

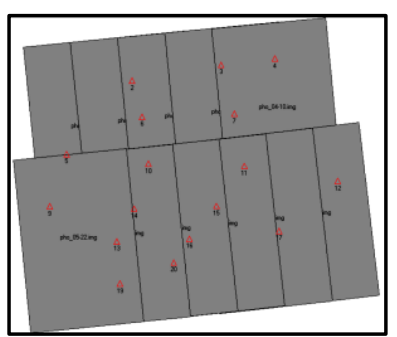

(b) - Second Block
Figure 1. Layout of the first and second block

\subsection{Methodology}

The proposed methodology considers that ISO needs accurate IOP values to improve the quality of refinement of direct EOP. Therefore, the IOP have to be estimated under flight condition. Additionally, due to the high correlation among parameters in the bundle adjustment, the new IOP values can model a parcel of inaccuracies of the direct determination of the EOP and the displacement of the collinearity condition. Considering this supposition, the traditional in-situ self-calibration of the imaging sensor using the physical model, as proposed by Brown, (1971) is used to compute a new set of the Interior Orientation Parameters. To perform this approach, a small subblock of images (five images in two strips) is extracted from the whole block. A minimum configuration of control points to fix the three-dimensional coordinates on the object space (2 Horizontal/Vertical and 1 Vertical) is required over the area of the small block. The values of the images' EOP from direct sensor orientation are included in the bundle adjustment as additional observations by weight constraint. The EOP values are weighted according to the nominal accuracies. A conventional set of tie points over the overlap areas should be defined and measurements. The RMSE of the residuals from the EOP weight constraint in the bundle adjustment will be used as the standard deviation of the direct EOP in the ISO approach.

Leica Photogrammetric Suite - LPS and UFPR Self Calibration Bundle Adjustment Software were used to perform the images measurement and the ISO experiments, respectively.

\section{RESULTS AND DISCUSSIONS}

Two main experiments were done to verify the proposed methodology to increase the performance of Integrated Sensor Orientation.

\subsection{Experiments using the first block}

Using the first block, a set of experiments was performed to verify the performance of the ISO approach to refine the values of EOP that were computed by direct sensor orientation, considering two main states (the quality of IOP and amount of control points required). The values of images' EOP from direct sensor orientation and calibrated IOP (focal length $=100.420$ $\mathrm{mm}$; coordinates of principal point $\mathbf{x}_{\mathbf{p}}=-0.135 \mathrm{~mm}$ and $\mathbf{y}_{\mathbf{p}}=$ $0.020 \mathrm{~mm}$ ) were provided by the photogrammetric company. All images don't have lens distortions. The block has 65 tie points over the overlap areas that were defined and measurement by automatic procedures.

A small sub block of images (five images in two strips, shown in Figure 2) was extracted from the whole first block to perform the in situ self-calibration. The sub block has 35 tie points, 3 control points ( 2 horizontal/vertical and 1 vertical).

The following precisions were considered for the measurements: 0.003 millimeters (half of pixel) for $\mathrm{x}$ and $\mathrm{y}$ image coordinates; two centimeters for 3D coordinates of the control points (from the GPS surveying); for the direct EOPs, nominal values were adopted (10 centimeters for positions and 18 seconds for Omega and Phi and 29 seconds for Kappa), considering the trajectory accuracy results in Post-Processing mode. The main results from the in situ self-calibration bundle adjustment are shown in Tables 1 .

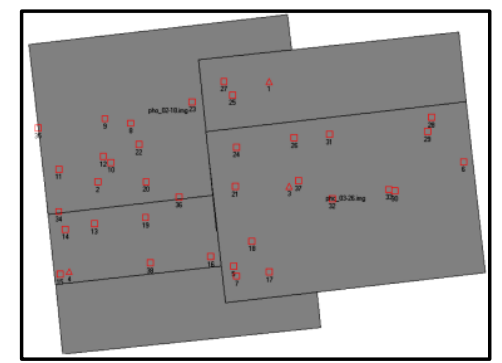

Figure 2. Layout of the sub block that was extracted from the first block

Table 2 shows the values of the root mean square errors (RMSE) of the measurements' residuals and the maximum 
values of the residuals that were computed from the obtained results in the self-calibration bundle adjustment. The reported values show that the majority of the RMSE are smaller than the a-priori precisions adopted for the measurements. Only the
RMSE value related to kappa is equal to the assumed precision. These aforementioned results and precisions of the IOP, shown in Table 1, confirm that the self-calibration bundle adjustment achieved acceptable precisions.

\begin{tabular}{c|c|c|c}
\hline \multicolumn{3}{c}{ IOP ESTIMATED IN SITU SELF- CALIBRATION } \\
\hline Experiment & $\mathbf{c}$ & $\mathbf{x}_{\mathbf{p}}$ & $\mathbf{y}_{\mathbf{p}}$ \\
& $\boldsymbol{\sigma}_{\mathbf{c}}(\mathbf{m m})$ & $\boldsymbol{\sigma}_{\mathbf{x p}}(\mathbf{m m})$ & $\boldsymbol{\sigma}_{\mathbf{y p}}(\mathbf{m m})$ \\
\hline First Sub & 100.437 & -0.119 & -0.003 \\
Block & 0.003 & 0.003 & 0.003 \\
\hline \multicolumn{3}{c}{$\mathrm{c}=$ Focal length; $\left(\mathbf{x}_{\mathbf{p}}, \mathbf{y}_{\mathbf{p}}\right)=$ Coordinates of principal point; } \\
$(\boldsymbol{\sigma})=$ Standard deviation.
\end{tabular}

Table 1. The interior orientation parameters (IOP) and its precisions estimated in selfcalibrations of the first sub block

\begin{tabular}{|c|c|c|c|c|c|c|c|c|c|c|}
\hline \multicolumn{11}{|c|}{ RESIDUALS ANALYSIS OF IN SITU SELF-CALIBRATION } \\
\hline \multicolumn{2}{|c|}{$\begin{array}{l}\text { RMSE of the residuals in } \\
\text { image coordinates (microns) }\end{array}$} & \multicolumn{3}{|c|}{$\begin{array}{l}\text { RMSE of residuals in control } \\
\text { point coordinates }(\mathrm{mm})\end{array}$} & \multicolumn{6}{|c|}{$\begin{array}{l}\text { RMSE of the residuals in EOPs } \\
\text { (centimeters and seconds) }\end{array}$} \\
\hline $\mathbf{x}$ & $\mathbf{y}$ & $\mathbf{X}$ & $\mathbf{Y}$ & $\mathbf{Z}$ & Xs & Ys & Zs & $\omega$ & $\varphi$ & $\chi$ \\
\hline 1 & 1 & 1 & 0 & 4 & 3 & 3 & 4 & 7 & 11 & 29 \\
\hline \multicolumn{11}{|c|}{ Maximum residuals } \\
\hline 3 & 3 & 1 & 0 & 5 & 5 & 6 & 7 & 11 & 14 & 43 \\
\hline
\end{tabular}

Table 2. Main results of the residuals analysis of the self-calibration bundle adjustment of the first sub block

The RMSE of the residuals of the coordinates of the positions of projection centers (Xs, Ys and Zs) are three times smaller than the a-priori adopted precision, as it can be seen in Table 2 . These results were expected, due to the direct correlations among parameters $\left(\mathrm{c}-\mathrm{Zs} ; \mathrm{x}_{\mathrm{p}}-\mathrm{Xs} ; \mathrm{y}_{\mathrm{p}}-\mathrm{Ys}\right)$. These correlations allow that the parameters related to focal length and coordinates of principal point can absorb some inaccuracies connected to the direct determination of the Xs, YS and Zs. On the other hand, there weren't additional parameters in collinearity equations directly correlated to the orientation parameters $(\omega, \varphi$, $\chi)$. Consequently, the residuals in Omega and Phi are approximately equal to a-priori adopted precisions. However, the residuals in Kappa are slightly bigger than the assumed precision, as can be seen in Table 2. These values of RMSE were used as the standard deviation of the direct EOP to perform the ISO experiment.

The lens distortion parameters were insignificant in the variance and covariance matrix. The estimated values of the focal length and coordinates of principal point are not equal to the values that were provided by photogrammetric company (the differences in focal length, $\mathbf{x}_{\mathbf{p}}$ and $\mathbf{y}_{\mathbf{p}}$ were $0.017 \mathrm{~mm}, 0.016 \mathrm{~mm}$ and $-0.023 \mathrm{~mm}$ respectively).

The next experiments were conducted to verify the performance of the ISO approach to refine the values of EOP of the first block. ISO experiments were performed using sets of IOP from photogrammetric company and from in situ calibration. In both conditions, the ISO experiments were performed without control points or using the same set of control points that was used in the in situ self-calibration ( 2 horizontal/vertical and 1 vertical).

A set of 83 check points was used as ground truth to verify the accuracies of the ISO experiments (analysis of 3D discrepancies). These check points are GCP and tie points over the first whole block area. The 3D coordinates of the tie points were computed in the conventional bundle adjustment (using 18 control points, only). In situ IOP were used in this bundle adjustment. As it was expected, the same results were obtained when IOP from the photogrammetric company were used.

\subsubsection{ISO experiments without control points}

Two experiments were performed. The first uses the IOP provided by the photogrammetric company and in the second one, the IOP, estimated by the in situ self-camera calibration, were used. The values of EOPS from the direct sensor orientation were included in the bundle adjustment as additional observations by weight constraint. In the first experiment, the EOP values were weighted according to the nominal precisions. The second experiment used the EOP precisions that were estimated in situ self-calibration. The main obtained results are shown in Tables 3 and 4.

\begin{tabular}{|c|c|c|c|c|c|c|c|c|c|c|c|}
\hline \multicolumn{12}{|c|}{ RESIDUALS ANALYSIS OF ISO EXPERIMENTS } \\
\hline \multicolumn{3}{|c|}{$\begin{array}{l}\text { RMSE of the Residuals in } \\
\text { image coordinates (microns) }\end{array}$} & \multicolumn{3}{|c|}{$\begin{array}{l}\text { RMSE of the residuals in } \\
\text { GCP coordinates }(\mathrm{mm})\end{array}$} & \multicolumn{6}{|c|}{$\begin{array}{l}\text { RMSE of the residuals in EOPs } \\
\text { (centimeters and seconds) }\end{array}$} \\
\hline & $\mathbf{x}$ & $\mathbf{y}$ & $\mathbf{X}$ & $\mathbf{Y}$ & $\mathbf{Z}$ & Xs & Ys & $\mathbf{Z s}$ & $\omega$ & $\varphi$ & $\chi$ \\
\hline IOP_Company & 1 & 1 & N/A & N/A & N/A & 9 & 8 & 4 & 25 & 22 & 25 \\
\hline IOP_In situ & 1 & 1 & N/A & N/A & N/A & 1 & 1 & 3 & 7 & 11 & 29 \\
\hline \multicolumn{12}{|c|}{ Maximum residuals } \\
\hline IOP_company & 5 & 3 & N/A & N/A & N/A & -15 & -13 & 12 & -32 & 40 & 43 \\
\hline IOP_In situ & 4 & 5 & N/A & N/A & N/A & -9 & -8 & 13 & -14 & 22 & 50 \\
\hline \multicolumn{12}{|c|}{ RMSE = Root mean square error } \\
\hline
\end{tabular}

Table 3. Main results of the residuals analysis of the ISO experiment of the first block without control points 


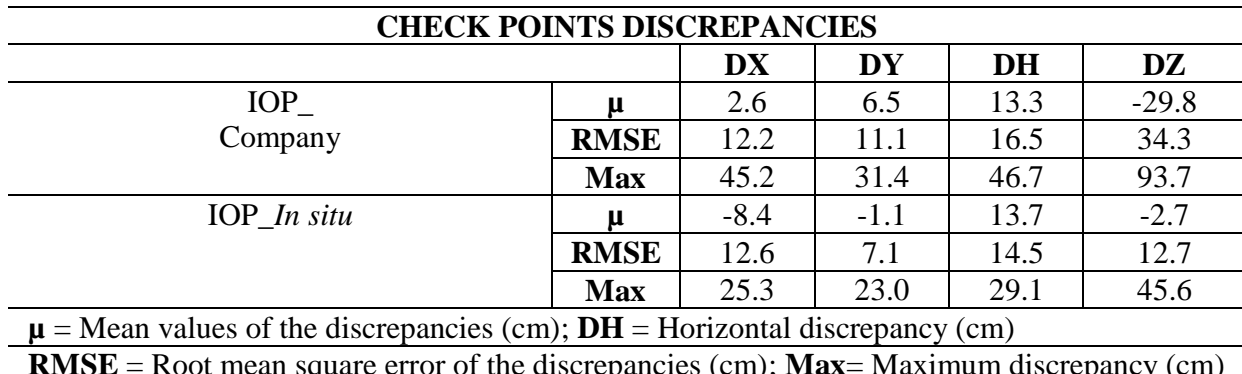

Table 4. Shows the results of discrepancies analysis performed in check points from the ISO experiment of the first block without control points.

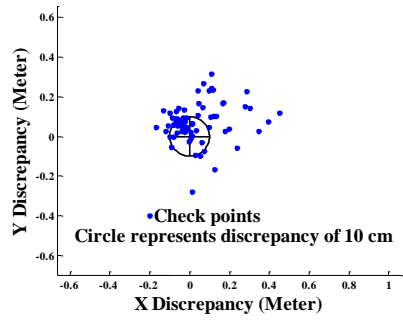

(a) - Horizontal discrepancies

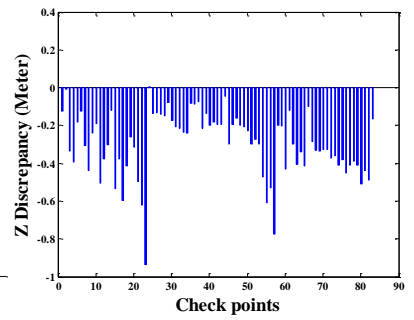

(b) - Vertical Discrepancies
Figure 3. Horizontal e vertical discrepancies of check points (first block) from ISO experiment without control points and IOP from photogrammetric company

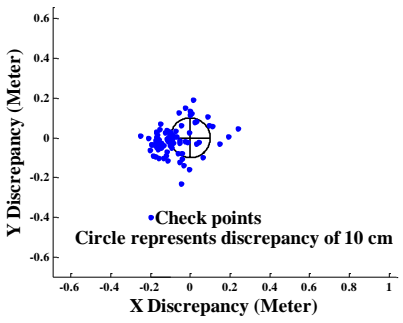

(a) - Horizontal discrepancies

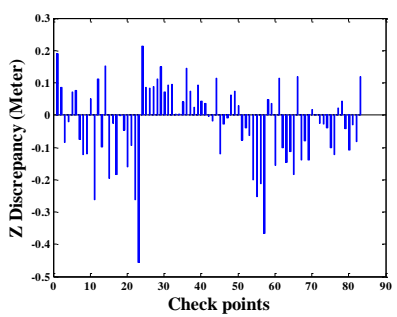

(b) - Vertical Discrepancies
Figure 4. Horizontal e vertical discrepancies of check points (first block) from ISO experiment without control points and IOP from in situ calibration
The obtained results from the two ISO experiments, reported in Tables 3 and 4, Figures 3 and 4, showed that the accuracies of the ISO experiment were improved significantly when the IOP and EOP precisions from in situ self-calibration were used. The vertical tendency found in the first ISO experiment was nearly eliminated in the second one. Additionally, considering the values of vertical RMSEs, the vertical accuracy increased 2.7 times. Albeit the RMSEs of the horizontal discrepancies in both experiments are nearly equal, the horizontal accuracy was improve in the second experiment, as can be seen in Figure 4

In conclusion; for the block the images and direct EOP used in this study, the proposed methodology improved the quality of ISO approach, performed, in principle, without control points.

\subsubsection{ISO experiments using control points}

The ISO experiments, previously discussed, were performed without control points. However the methodology that was proposed to improve the horizontal and vertical accuracies of the ISO approach requires at least three control points (two horizontal/vertical and one vertical) to estimate new IOP under flight conditions. Therefore, the same set of control points should be included in the ISO approach. To verify this proposal, the same experiments, previously studied, were performed using the set of control points. The obtained results from the experiments are shown Tables 5 and 6, Figures 5 and 6.

\begin{tabular}{|c|c|c|c|c|c|c|c|c|c|c|c|}
\hline \multicolumn{12}{|c|}{ RESIDUALS ANALYSIS OF ISO EXPERIMENTS } \\
\hline \multicolumn{3}{|c|}{$\begin{array}{l}\text { RMSE of the Residuals in } \\
\text { image coordinates (microns) }\end{array}$} & \multicolumn{3}{|c|}{$\begin{array}{l}\text { RMSE of the residuals in } \\
\text { GCP coordinates }(\mathrm{mm})\end{array}$} & \multicolumn{6}{|c|}{$\begin{array}{l}\text { RMSE of the residuals in EOPs } \\
\text { (centimeters and seconds) }\end{array}$} \\
\hline & $\mathbf{x}$ & $\mathbf{y}$ & $\mathbf{X}$ & $\mathbf{Y}$ & $\mathbf{Z}$ & Xs & Ys & $\mathbf{Z s}$ & $\omega$ & $\varphi$ & $\chi$ \\
\hline IOP_Company & 1 & 1 & 7 & 3 & 12 & 9 & 8 & 8 & 25 & 29 & 22 \\
\hline IOP_In situ & 1 & 2 & 6 & 6 & 10 & 2 & 1 & 3 & 7 & 14 & 29 \\
\hline \multicolumn{12}{|c|}{ Maximum residuals } \\
\hline IOP_company & 5 & 6 & -7 & -5 & 19 & -18 & 13 & -13 & -40 & 50 & 36 \\
\hline IOP_In situ & 5 & 7 & 7 & 4 & 13 & -3 & -4 & 7 & -18 & 25 & 54 \\
\hline \multicolumn{12}{|c|}{ RMSE = Root mean square error } \\
\hline
\end{tabular}

Table 5. Main results of the residuals analysis of the ISO experiment of the first block with control points 


\begin{tabular}{c|c|c|c|c|c}
\hline \multicolumn{7}{c}{ CHECK POINTS DISCREPANCIES } \\
\hline \multirow{2}{*}{$\begin{array}{c}\text { IOP_ } \\
\text { Company }\end{array}$} & $\boldsymbol{\mu}$ & $\mathbf{D X}$ & $\mathbf{D Y}$ & $\mathbf{D H}$ & $\mathbf{D Z}$ \\
\cline { 2 - 6 } & $\mathbf{R M S E}$ & 6.8 & 0.5 & 7.5 & -15.9 \\
\cline { 2 - 6 } & $\mathbf{M a x}$ & 24.6 & 23.8 & 28.6 & 65.3 \\
\hline \multirow{2}{*}{ IOP_In situ } & $\boldsymbol{\mu}$ & -1.1 & -0.8 & 7.9 & -1.7 \\
\cline { 2 - 6 } & $\mathbf{R M S E}$ & 7.5 & 5.5 & 9.3 & 9.7 \\
\cline { 2 - 6 } & $\mathbf{M a x}$ & 23.6 & 17.8 & 24.1 & 32.6
\end{tabular}

$\boldsymbol{\mu}=$ Mean values of the discrepancies (cm); $\mathbf{D H}=$ Horizontal discrepancy $(\mathrm{cm})$

RMSE = Root mean square error of the discrepancies $(\mathrm{cm}) ;$ Max= Maximum discrepancy $(\mathrm{cm})$

Table 6. Shows the results of discrepancies analysis performed in check points from the ISO experiment of the first block with control points

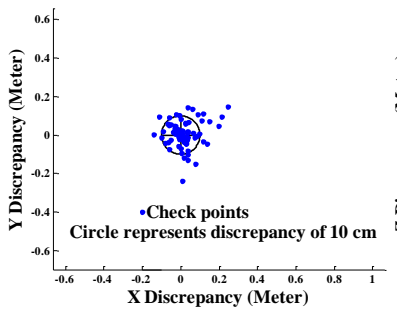

(a) - Horizontal discrepancies

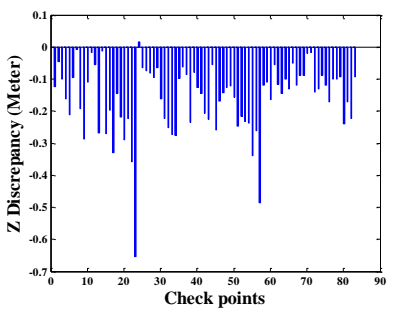

(b) - Vertical Discrepancies
Figure 5. Horizontal e vertical discrepancies of check points (first block) from ISO experiment with control points and IOP from photogrammetric company

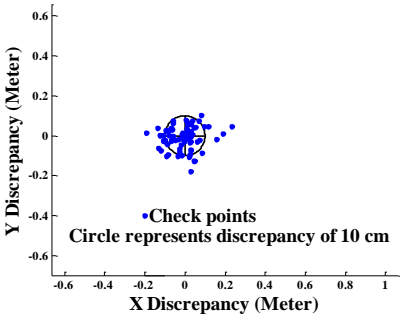

(a) - Horizontal discrepancies

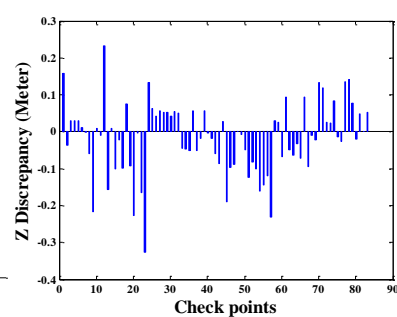

(b) - Vertical Discrepancies
Figure 6. Horizontal e vertical discrepancies of check points (first block) from ISO experiment with control points and IOP from in situ calibration

The performance of the ISO approach with a set of control points has improved, as can be seen in the obtained results reported in Tables 5 and 6 . However, the procedure was not able to fix the systematic vertical tendency, found in the first ISO experiment when the IOP from the photogrammetric company were used. Comparing results from check points discrepancies between ISO experiments with control points and ISO experiments without control points, reported in Figures 5 and 6 and Figures 3 and 4 respectively, it is possible to note that vertical and horizontal accuracies in the ISO approach with control points was proportionally increased. Based on the horizontal and vertical RMSE values, computed with check point discrepancies, the ISO approach using control points and IOP from photogrammetric company increased its horizontal and vertical accuracies in $57 \%$ and $56 \%$ respectively. In ISO approach, using control points and IOP from in sit self- calibration, the horizontal and vertical accuracies increased respectively $64 \%$ and $76 \%$.

Although the use of the set of control points increased the performance of the ISO approaches, better accuracies were obtained when IOP and EOP precisions from in situ selfcalibration were used. Based on the computed values of RMSE from the check point discrepancies, the horizontal and vertical accuracies increased $1 \%$ and $50 \%$ respectively. Additionally, the systematic vertical tendency was fixed.

In conclusion; for the block of images and dataset of direct EOP used in this study, the ISO approach achieved horizontal and vertical accuracies nearly to one image pixel of resolution on the ground (GSD) when control points and IOP from in situ calibration were used.

\subsection{Experiments using the second block}

Due to the similarity of the obtained results from the experiments and discussions, only main results of the next experiments were shown and discussed. The main objective of the next experiments is to show the performance of the proposed methodology for another similar block of images.

Using the same steps of work, previously discussed on the first block, one small sub block of images (5 images in two strips) was extracted from the whole second block. As the first block, the in situ self-calibration was performed. Only the focal length and coordinates of principal point were considered. The estimated values are: focal length $(\mathrm{c})=100.439 \mathrm{~mm}, \boldsymbol{\sigma}_{\mathrm{c}}=0.004$ $\mathrm{mm}$; coordinates of principal point $\left(\mathbf{x}_{\mathbf{p}}\right)=-0.113 \mathrm{~mm}, \boldsymbol{\sigma}_{\mathbf{x p}}=$ $0.004 \mathrm{~mm} ;\left(\mathbf{y}_{\mathbf{p}}\right)=-0.011 \mathrm{~mm}, \boldsymbol{\sigma}_{\mathbf{y p}}=0.004 \mathrm{~mm}$. Using the residuals of the EOP weight constraint in the self-calibration bundle adjustment, their RMSE were computed to use them as the standard deviation of the direct EOP in the next ISO approach. The RMSE values are: $(\mathrm{Xs})=2.9 \mathrm{~cm}$; $(\mathrm{Ys})=3.6 \mathrm{~cm}$; $(\mathrm{Zs})=4.8 \mathrm{~cm} ;(\boldsymbol{\omega})=7$ seconds; $(\boldsymbol{\varphi})=7$ seconds; $(\boldsymbol{\chi})=90$ seconds. The entire second block has 17 signalized control points and 65 tie points over the overlap areas that were defined and measurement by automatic procedures. Using the same procedures previously performed and discussed in the first block, the ISO experiments were performed. The obtained results from the ISO approach using control points and two different sets of IOP are reported in Tables 7 and 8 and Figures 7 and 8. 


\begin{tabular}{|c|c|c|c|c|c|c|c|c|c|c|c|}
\hline \multicolumn{12}{|c|}{ RESIDUALS ANALYSIS OF ISO EXPERIMENTS } \\
\hline \multicolumn{3}{|c|}{$\begin{array}{l}\text { RMSE of the Residuals in } \\
\text { image coordinates (microns) }\end{array}$} & \multicolumn{3}{|c|}{$\begin{array}{l}\text { RMSE of the residuals in } \\
\text { GCP coordinates }(\mathrm{mm})\end{array}$} & \multicolumn{6}{|c|}{$\begin{array}{l}\text { RMSE of the residuals in EOPs } \\
\text { (centimeters and seconds) }\end{array}$} \\
\hline & $\mathbf{x}$ & $\mathbf{y}$ & $\mathbf{X}$ & $\mathbf{Y}$ & $\mathbf{Z}$ & $\mathbf{X s}$ & Ys & Zs & $\omega$ & $\varphi$ & $\chi$ \\
\hline IOP_Company & 1 & 1 & 3 & 4 & 12 & 12 & 12 & 8 & 40 & 43 & 97 \\
\hline IOP_In situ & 1 & 1 & 4 & 1 & 4 & 1 & 2 & 3 & 7 & 11 & 108 \\
\hline \multicolumn{12}{|c|}{ Maximum residuals } \\
\hline IOP_company & 7 & 6 & -4 & 5 & 18 & 17 & 17 & -12 & -50 & 65 & 141 \\
\hline IOP_In situ & 3 & 4 & 5 & -2 & 6 & 2 & -4 & -6 & 14 & 18 & -173 \\
\hline
\end{tabular}

Table 7. Main results of the residuals analysis of the ISO experiment of the second block with control points

\begin{tabular}{c|c|c|c|c|c}
\hline \multicolumn{7}{c}{ CHECK POINTS DISCREPANCIES } & \multicolumn{1}{c}{ DX } \\
\hline \multirow{2}{*}{$\begin{array}{c}\text { IOP_ } \\
\text { Company }\end{array}$} & $\boldsymbol{\mu}$ & -4.1 & -0.1 & 11.5 & -41.1 \\
\cline { 2 - 6 } & $\mathbf{R M S E}$ & 10.1 & 10.4 & 14.5 & 46.0 \\
\cline { 2 - 6 } & $\mathbf{M a x}$ & -39.3 & 33.6 & 43.3 & -82.9 \\
\hline \multirow{2}{*}{ IOP_In situ } & $\boldsymbol{\mu}$ & -3.8 & -2.3 & 7.1 & -1.0 \\
\cline { 2 - 6 } & $\mathbf{R M S E}$ & 7.3 & 4.9 & 8.8 & 11.1 \\
\cline { 2 - 6 } & $\mathbf{M a x}$ & -24.2 & -13.7 & 27.1 & 32.7 \\
\hline
\end{tabular}

$\boldsymbol{\mu}=$ Mean values of the discrepancies $(\mathrm{cm}) ; \mathbf{D H}=$ Horizontal discrepancy $(\mathrm{cm})$

RMSE $=$ Root mean square error of the discrepancies $(\mathrm{cm}) ;$ Max= Maximum discrepancy $(\mathrm{cm})$

Table 8. Shows the results of discrepancies analysis performed in check points of the ISO experiment of the second block with control points

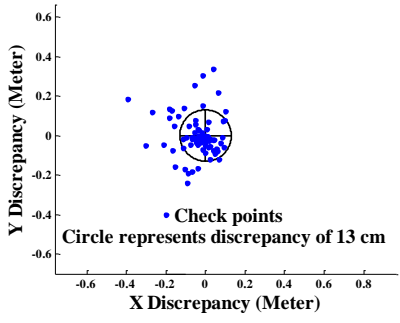

(a) - Horizontal discrepancies

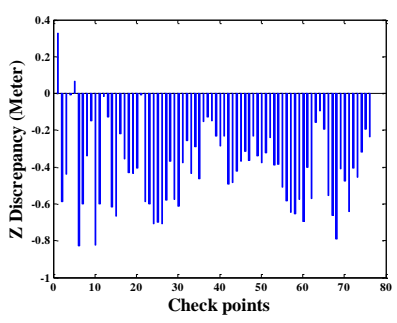

(b) - Vertical Discrepancies
Figure 7. Horizontal e vertical discrepancies of check points (second block) from ISO experiment with control points and IOP from photogrammetric company

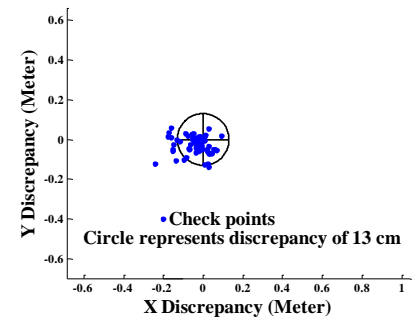

(a) - Horizontal discrepancies

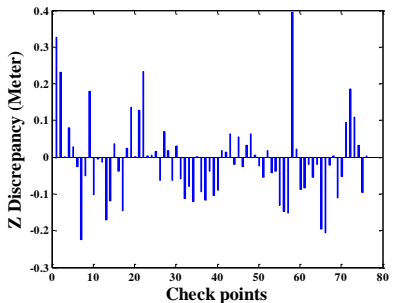

(b) - Vertical Discrepancies
Figure 8. Horizontal e vertical discrepancies of check points (second block) from ISO experiment with control points and IOP from in situ calibration

The obtained results from the two experiments of ISO approach with control points, reported in Tables 7 and 8 , confirm the main results and conclusions that were discussed on the ISO study using the first block of images and confirm the efficiency of the proposed methodology to increase the performance of ISO approach. Comparing the obtained results from the two ISO experiments, reported in Tables 7 and 8, Figures 7 and 8, it can be noted that the ISO approach using a set of control points (two horizontal/vertical and one vertical) and the IOP from in situ self-calibration increases significantly the accuracies of 3D point intersection. Based on the computed values of RMSE of the check point discrepancies, the horizontal and vertical accuracies improved nearly 0.6 and 4 times. Additionally, the systematic vertical tendency was fixed. Again, the proposed methodology improved the performance of the ISO approach achieving horizontal and vertical accuracies nearly to one image pixel of resolution on the ground (GSD).

\section{CONCLUSION AND OUTLOOK}

The paper showed the results of an empiric study that was performed to increase the performance of Integrated Sensor Orientation (ISO) approach based on the hypothesis that IOP estimated under flight condition improve the ISO quality. To perform the study, two small blocks of images with different configuration were used. In the first block, the strips were flown in directions N-S and S-N and the second in W-E and E-W. Small sub blocks of images (five images in two strips) were extracted from the entire blocks to perform the in situ selfcalibrations. A minimum configuration of control points over the area of the small sub block was used to fix the threedimensional coordinates on the object space (2 Horizontal/Vertical and 1 Vertical). The set of the direct EOP of the images from the direct sensor orientation was included in 
the bundle adjustment as additional observations by weight constraint. The RMSE of the residuals from these additional observations were used as the standard deviation of the direct EOP in ISO approach. The new IOP from the in situ selfcalibration with the set of control points were also used in the ISO approach. From the results of the performed experiments, the main conclusions are drawn:

- The methodology proposed to increase the performance of Integrated Sensor Orientation (ISO) approach requires Interior Orientation Parameters (IOP) and standard deviation of direct EOP under flight condition. In situ self-calibrations approach was used to estimate these values. A small sub block of images (5 images in two strips) extracted from the entire block was used;

- Two small blocks of images were used to perform the experiments of ISO approaches. The experiments of ISO approach using IOP and standard deviation of direct EOP under flight condition improved significantly the vertical and horizontal accuracies of the $3 \mathrm{D}$ point intersection. To achieve horizontal and vertical accuracies nearly to one image pixel of resolution on the ground (GSD), the set of control points (2 Horizontal/Vertical and 1 Vertical) should be included;

- The ISO approach without control points was investigated. However, using this configuration the horizontal and vertical accuracies in $3 \mathrm{D}$ point intersection were close to 1.5 image pixel of resolution on the ground (GSD);

- The proposed methodology to increase the ISO approach achieved better results in the performed experiments. However, due to the small dimension of the two used blocks of images, the procedure must be evaluated in larger block to confirm its performance.

For further works, it will focus on verification of the proposed methodology to increase the ISO performance using blocks of images with more strips and images. The experiments will be conducted to investigate the improvement de horizontal and vertical accuracies of $3 \mathrm{D}$ photogrammetric point intersection in the ISO approach using IOP and standard deviation of direct EOP under flight condition.

\section{ACKNOWLEDGMENTS}

We would like to thank the two Brazilian governmental agencies CNPq (The National Council for Scientific and Technologic Development) and CAPES (The Coordinating Agency for Advanced Training of High-Level Personnel) for their financial support of this research and TOPOCART (Surveying Engineering and Aero Mapping Company) to provide the necessaries material to develop this study.

\section{REFERENCES}

Brown, D., 1971. Close range camera calibration, Photogrammetric Engineering, 37(8), 855-866.
Colomina, I., 1999. GPS, INS and aerial triangulation: What is the best way for the operational determination of photogrammetric image orientation? IAPRS (32) 3-2W5, pp.121-130.

CRAMER, M., 1999. Direct geocoding - is aerial triangulation obsolete? in: Fritsch D., Spiller R. (Eds.), Photogrammetric Week' 99, pp. 59-70.

Cramer, M., and D. Stallmann, 2001. OEePE test on integrated sensor orientation - IFP results and experience, Proceedings of the OEEPE Workshop, Integrated Sensor Orientation, Hanover, Germany, 17-18 September.

Cramer, M. and Stallmann, D., 2002. System Calibration for Direct Georeferencing, International Archives of Photogrammetry, Remote Sensing and Spatial Information Sciences, 34(3/A), 79-84.

Ip, A., El-Sheimy, N., Mostafa, M., 2007. Performance analysis of integrated sensor orientation, Photogrammetric Eng. Remote Sens., vol. 73, no. 1, 89-97.

JACOBSEN, K., 2000. Combined Bundle Block Adjustment versus Sensor Orientation, ASPRS Annual Convention 2000, Washington D.C.

Jacobsen, K., Wegmann, H., 2002. Dependencies and problems of Direct Sensor Orientation. "Integrated Sensor Orientation", Integrated Sensor Orientation, Test Report and Workshop Proceedings (C. Heipke, K. Jacobsen, and H. Wegmann, editors), OEEPE Official Publication No. 43, 73-84.

Heipke, C., K. Jacobsen, AND H. Wegmann, 2002. Analysis of the Results of the OEEPE Test "Integrated Sensor Orientation", Integrated Sensor Orientation, Test Report and Workshop Proceedings (C. Heipke, K. Jacobsen, and H. Wegmann, editors), OEEPE Official Publication No. 43, 31-49.

Honkavaara, E., Ilves, R., JaAkkola, J., 2003. Practical Results of GPS/IMU/camera System Calibration. In: Proc. Of Workshop: Theory, Technology and Realities of Inertial/GPS/Sensor Orientation, ISPRS WG I/5, Barcelona, CD-ROM, $10 \mathrm{p}$.

Тотн, C., 1999. Experiences with frame CCD arrays and direct georeferencing, in: Fritsch D., Spiller R. (Eds.), Photogrammetric Week'99, pp. 95-107.

Wegmann, H., 2002. Image Orientation by Combined (A)AT with GPS and IMU. In: Proceedings of ISPRS Commission I Mid-term Symposium, Nov. 2002, Denver, Colorado, USA.

YASTIKLI, N., JACOBSEN K., 2005. Influence of System Calibration on Direct Sensor Orientation. Photogrammetric Engineering and Remote Sensing, Vol. 71 No 5, May 2005, 629-633. 